\title{
Contrast-enhanced ultrasound for ovary assessment in a murine model: preliminary findings on the protective role of a gonadotropin-releasing hormone analogue from chemotherapy-induced ovarian damage
}

\author{
Massimo Venturini ${ }^{1,2^{*}}$, Alice Bergamini ${ }^{3}$, Laura Perani ${ }^{2}$, Ana Maria Sanchez ${ }^{3}$, Elena Giulia Rossi ${ }^{3}$, Anna Colarieti $^{1}$, \\ Micaela Petrone ${ }^{3}$, Francesco De Cobelli ${ }^{1,4}$, Alessandro Del Maschio ${ }^{1,4}$, Paola Viganò ${ }^{3}$, Giorgia Mangili ${ }^{3}$, \\ Massimo Candiani, ${ }^{3,4}$, Carlo Tacchetti ${ }^{2,4}$ and Antonio Esposito ${ }^{1,2,4}$
}

\begin{abstract}
The prolonged, gonadotoxic effect of chemotherapy can finally lead to infertility in female cancer survivors. There is controversial evidence regarding the protective role of gonadotropin-releasing hormone analogue (GnRH-a) on chemotherapy-induced ovarian damage. In the present study on a murine model, ultrasound (US) and contrastenhanced US (CEUS) were firstly used to characterise ovarian glands in normal conditions to validate a preclinical model. In addition, preliminary findings were obtained on anatomical and vascular ovarian changes induced by GnRH-a based on decapeptyl administration. Ovaries were accurately assessed with US and CEUS in a murine model placed in prone position, providing quantitative and reproducible information. Ovaries were identified in 40/40 cases and CEUS analysis was successfully performed in 20/20 cases with 100\% technical success. A statistically significant increase of the diameter of the dominant follicle at US and a statistically significant reduced vascularisation at CEUS in decapeptyl-treated mice compared to untreated control mice were recorded. Further studies using US and CEUS in the murine model combining GnRH-a and chemotherapeutic agents will be needed to obtain more translational information useful for clinical practice.
\end{abstract}

Keywords: Disease models (animal), Gonadotropin-releasing hormone, Infertility (female), Ovary, Ultrasonography

\section{Key points}

- Ovaries, despite their limited size, can be easily identified at US in the murine model as oval, hypoechoic images with net margins, with diameter usually variable from 1 to $2.5 \mathrm{~mm}$, located inferiorly and laterally from the lower pole of the corresponding kidney.

\footnotetext{
* Correspondence: venturini.massimo@hsr.it

${ }^{1}$ Department of Radiology, San Raffaele Scientific Institute, Milan, Italy

${ }^{2}$ Experimental Imaging Center, San Raffaele Scientific Institute, Milan, Italy

Full list of author information is available at the end of the article
}

- At US, the diameter of the dominant follicle increased significantly in mice treated with GnRH-a (decapeptyl) mice compared to control untreated mice.

- At CEUS, a reduced vascularisation was found in mice treated with GnRH-a (decapeptyl) compared to control.

\section{Background}

Prognostic improvement obtained in cancer treatment over the last decades emphasises the long-term side effects of chemotherapy. During the last decades, the improvement in prognosis obtained in the treatment of 
most tumours emphasises the long-term collateral effects of chemotherapy. Gonadotoxicity and fertility reduction are emerging as a crucial challenge for physicians, and this is particularly true in young female cancer survivors [1,2]. Chemotherapy induces ovarian toxicity through primordial follicular apoptosis, follicular burn-out, stromal fibrosis, and alteration of the vasculature structure [3]. Fertility preservation techniques have become increasingly important to prevent ovarian damage, and the issue of preserving fertility before starting chemotherapy has been addressed by clinical oncologists $[1,4]$.

Gonadotropin-releasing hormone analogues (GnRH-a) are used in clinical practice, even though the protective mechanisms preventing ovarian insufficiency are still controversial [5]. Translational studies on ovarian glands in mice using ultrasound (US) and contrastenhanced US (CEUS) have been mainly performed to validate models of ovarian cancer [6], while there is a paucity of experimental studies regarding imaging of normal ovaries. The lack of a well-established US technique for murine ovary detectability and analysis is a gap which has to be filled to build a reproducible preclinical model.

The first aim of this experimental study was to assess the technical aspects and feasibility of US and CEUS to characterise ovaries in normal conditions to validate a preclinical model. The second aim was to obtain preliminary findings on $\mathrm{GnRH}$-a-induced ovarian anatomical and vascular changes assessed by US (dominant follicle diameter) and CEUS (vascular perfusion parameters), respectively.

\section{Methods}

\section{Study design}

This is an experimental, single-centre study in a murine model comparing two groups of female $\mathrm{BALB} / \mathrm{c}$ mice, a well-known, typical breed of mice used in biomedical research. The mice were treated with subcutaneous injection of a GnRH-a, $100 \mu \mathrm{L}$ of Decapeptyl SR (Ipsen, Milan, Italy) equivalent to $4.45 \mathrm{mg} / \mathrm{kg} \quad(n=10$, experimental group) or of $100 \mu \mathrm{L}$ of Dulbecco's phosphate-buffered saline (Euroclone SPA, Milan, Italy). The two groups were compared to preliminarily assess gonadotropin down-regulation using US and CEUS.

\section{Mice}

The study was approved by the Animal Care and Use Committees of our hospital. Animals were housed in the Institutes' Animal Care Facilities, according to international standards. BALB/c female mice (14 weeks old) were housed in air-conditioned, pathogen-free, lightcontrolled animal facilities of the experimental imaging centre of our hospital.

\section{Animal preparation}

Mice were anaesthetised with isoflurane (flurane; Isoba, Schering-Plough, San Diego, CA, USA), $4 \%$ in oxygen for induction, $2 \%$ for maintenance at a rate of $1 \mathrm{~L} / \mathrm{min}$. After the mice were completely anaesthetised, the tail veins were catheterised using 10-cm-long, 27-gauge polyethylene tubing. Mice were positioned in prone position on a MousePad (THM150 MousePad part of the VisualSonics Vevo Integrated Rail System III, Toronto, Canada) equipped with an integrated heater and electrocardiography electrodes. The four legs were secured to electrocardiography pads, with mediation of electrode cream to allow a continuous monitoring of all vital parameters: temperature, respiration rate, cardiac frequency and electrocardiogram. Body temperature was monitored with a rectal probe thermometer. A depilatory cream was used to remove fur from the region of interest, and prewarmed ultrasound gel was used as a coupling agent between the ultrasound probe and the skin.

\section{US and CEUS examinations}

B-mode US scans were performed with Vevo 2100 ultrasonographic system high-frequency linear probes, $40 \mathrm{MHz}$ for US and $20 \mathrm{MHz}$ for CEUS (FUJIFILM VisualSonics Inc., Toronto, Canada). All the examinations were performed by the same biologist with more than 5 years of experience in experimental US. All the US and CEUS acquisition data as well as the images were reviewed by a radiologist with more than 5 years of experience in preclinical US and more than 20 years of experience in clinical US and CEUS. Using a mechanical arm that keeps the $40 \mathrm{MHz}$ linear probe fixed, both ovaries were ultrasonically assessed as oval hypoechoic structures, with net margins, vascularised, located inferiorly and laterally from the lower pole of the corresponding kidney (Fig. 1a, b). Ovary major diameter and dominant follicle diameter were major parameters under consideration. After the high-resolution definition of both ovarian glands obtained with the $40 \mathrm{MHz}$ linear probe, the $20 \mathrm{MHz}$ linear probe was mounted on the mechanical arm to perform CEUS, choosing the ovarian gland better identifiable at the US examination. The probe was fixed on the railing system to maintain the acoustic focus at the centre of the ovary at the level of the largest transverse cross section. CEUS studies were performed during intravenous bolus injection of Vevo MicroMarker (Bracco, Geneva, Switzerland). The contrast agent was injected using a syringe pump (Pump 11 Elite, Harvard Apparatus, Holliston, MA, USA) as a bolus via tail vein catheter at a rate of $0.750 \mathrm{~mL} / \mathrm{min}$ for $5 \mathrm{~s}\left(50 \mu \mathrm{L}\right.$ bolus, $3.5 \times 10^{7}$ microbubbles $)$ in $4 \mathrm{~s}$. During imaging, body temperature was maintained between 36 and $38{ }^{\circ} \mathrm{C}$. The data acquisition was started immediately after contrast agent administration, and cine 


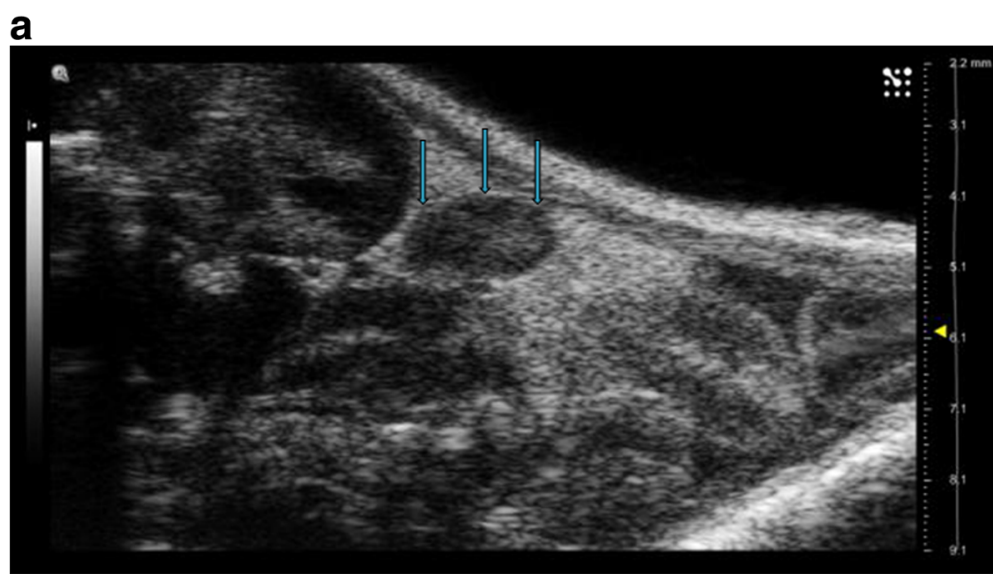

b

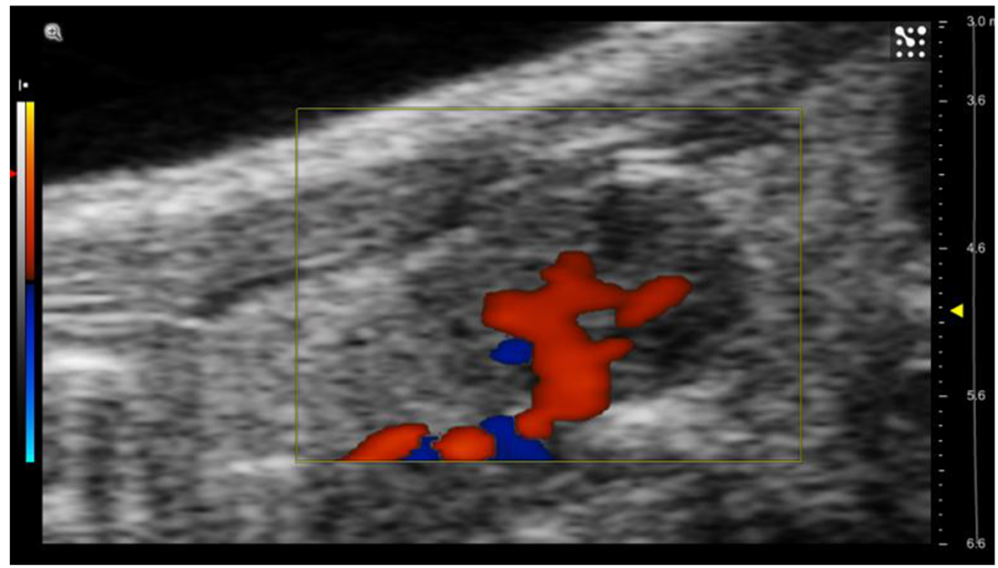

Fig. 1 a US examination of the mouse placed in prone position shows the right ovary as an oval, hypoechoic nodule with net margin located inferiorly and laterally to the lower pole of the corresponding kidney (arrows). b Colour Doppler examination well defines vascularisation and particularly the ovarian artery course

loops of contrast wash-in were acquired for $55 \mathrm{~s}$. To reduce variability, the used US parameters were the following: power 4\%; dynamic range $40 \mathrm{~dB}$; frequency $18 \mathrm{MHz}$; frame rate 36; contrast gain $49 \mathrm{~dB}$; gate 4 .

To ensure the quality of the CEUS examination, the ovarian enhancement was assessed during contrast administration, using a side-by-side technique, i.e. in real-time simultaneous visualisation of both the baseline US images demonstrating the ovarian gland as a hypoechoic structure and the CEUS dynamic acquisition showing the ovary progressively becoming hyperechoic (Fig. 2). This assessment was performed at three different time points (day 0 , day 10 , and day 20 ).

\section{CEUS images analysis}

Recorded cine loops were processed off-line for time-intensity curve analysis using VevoCQ Advanced Contrast Quantification Software (FUJIFILM VisualSonics). A region of interest was drawn along the perimeter of the ovary. The time-intensity curve for each imaging protocol was plotted, and a mathematical equation model [7] was used to fit the contrast uptake time-intensity curve. Perfusion parameters extracted from the fitted model were the following:

- Peak enhancement, defined as the difference between the maximum amplitude in the curve and the baseline level, and is thus indicative of relative blood volume

- Wash-in rate, defined as the maximum slope of the fitted curve, in the wash-in phase

- Time to peak, which expresses the time in seconds from the time origin to the peak of the fitted curve

- Rise time, defined as the time from the instant at which the maximum slope tangent intersects the $x$-axis to the peak of the fitted curve, and is thus independent of the time origin

- Area under the curve, defined as area under the curve to infinite time 


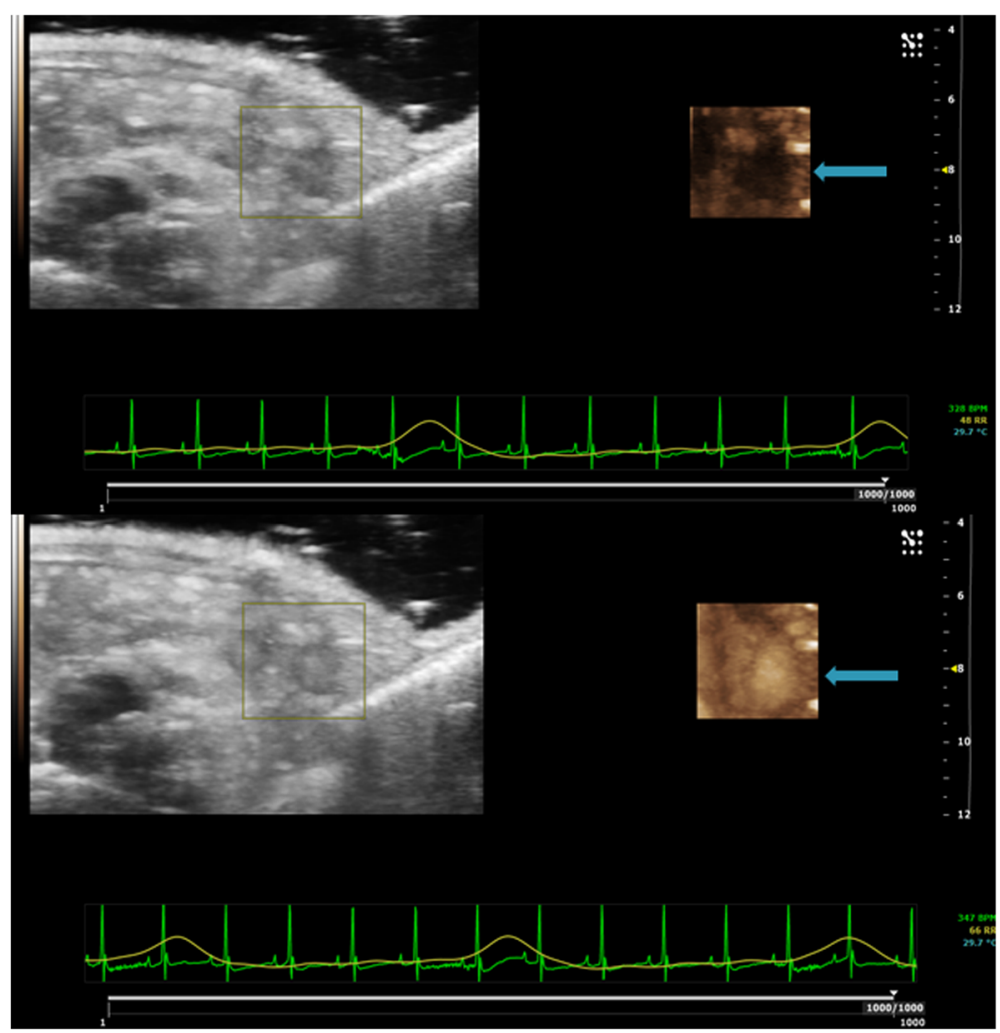

Fig. 2 CEUS dynamic acquisition using a side-by-side visualisation (see text) shows the hypoechoic ovary becoming progressively hyperechoic due to the contrast enhancement (arrows)

- Mean transit time, defined as the average time required for the contrast agent to pass through the region of interest

- Perfusion index, defined as area under the curve divided by mean transit time

- Wash-in perfusion index, defined as wash-in area under the curve divided by rise time

- Wash-in area under the curve, defined as the area under the curve from starting enhancement to peak enhancement.

\section{Variable analysis}

Technical success, defined as ovaries identification with US and CEUS analysis reliability performed with Advanced Contrast Quantification Software, was preliminarily assessed. Subsequently, comparing decapeptyltreated mice with untreated mice (controls) at day 0 , day 10 , and day 20 , the following parameters were investigated: ovary major diameter and dominant follicle major diameter (using US); all the perfusion parameters listed in the previous paragraph (using CEUS).

\section{Statistical analysis}

Statistical analysis was performed by $t$ test with twotailed distribution and two-sample unequal variance
(GraphPad Prism software, version 5.04). The differences were considered significant when the $p$ value was lower than 0.05. Data are presented as means \pm standard deviation.

\section{Results}

Feasibility of US and CEUS

Both ovarian glands were visualised and accurately evaluated at US in all cases at the three different time points. In all cases the ovaries were easily identified at US inferiorly and laterally from the lower pole of the corresponding kidney as oval, hypoechoic nodules with net margins and with a diameter variable from 1 to $2.5 \mathrm{~mm}$. Ovaries were identified in $40 / 40$ cases with $100 \%$ technical success at day 0 , day 10 and day 20. CEUS analysis with Advanced Contrast Quantification Software was successfully performed in $20 / 20$ cases with $100 \%$ technical success at day 0, day 10 and day 20.

\section{Comparison between decapeptyl-treated mice and control untreated mice}

Considering US parameters, no significant differences in major diameter were found between decapeptyl-treated mice and controls (Table 1). The diameter of the dominant follicle increased significantly in decapeptyl-treated 


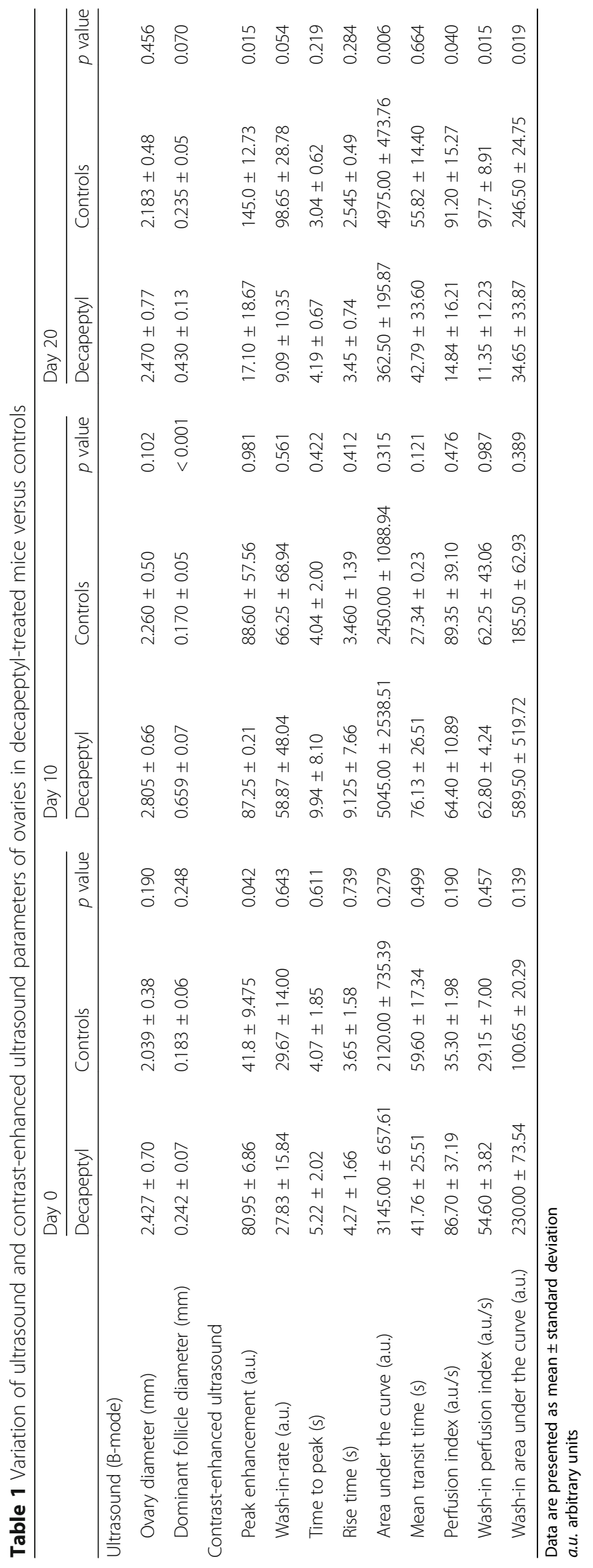


mice compared to control untreated mice (follicles $>$ $0.4 \mathrm{~mm}$ versus follicles $<0.2 \mathrm{~mm}$ ) at day 10 and day 20 (Table 1, Fig. 3). In the control group no significant variation in this parameter was observed. CEUS of the ovary better identifiable at the preliminary US was successfully performed in all cases, with real-time demonstration of progressive enhancement of the ovarian gland during contrast agent administration. Considering CEUS parameters, a progressive reduction in vascular perfusion was found in decapeptyl-treated mice compared with controls (Fig. 4a). At day 20, a significant reduction in the peak enhancement and wash-in area under the curve was found in decapeptyl-treated mice compared with control untreated mice (Fig. 4b).

\section{Discussion}

Chemotherapy-induced ovarian damage may be variable, leading to transient/permanent amenorrhea, infertility or early menopause $[8,9]$. In the past the use of temporary ovarian suppression with luteinising hormone-releasing hormone analogues [10] and oral contraceptives during chemotherapy has been recommended to preserve fertility [11]. Fertility preservation techniques are becoming a fundamental step of the therapeutic management.
Nowadays, embryo/oocyte cryopreservation [12] may be an alternative, although cost and feasibility are unsuitable for all patients. On the other hand, the role of $\mathrm{GnRH}-\mathrm{a}$ in preventing ovarian damage still remains unclear and controversial.

While single-arm and retrospective studies demonstrated encouraging findings [13, 14], randomised trial data have shown conflicting results $[15,16]$, partially explained by heterogeneous study populations, variable procedures and different chemotherapeutic treatment regimens.

In this preliminary experimental study, the first endpoint was to evaluate with US and CEUS ovaries in normal conditions to obtain a valid preclinical model, and the second end-point was to preliminarily verify the GnRH-a action to perform further prospective studies with chemotherapeutic agents. Ovaries, despite their tiny dimensions (1-2.5 $\mathrm{mm}$ ), were assessed with US and CEUS: US identification and CEUS analysis of ovaries were successfully performed in all cases $(100 \%$, full technical success). Their peculiar position (inferiorly and laterally from the lower pole of the corresponding kidney) and their clear US definition (oval morphology, net margins, hypoechoic structure, vascularisation representation)

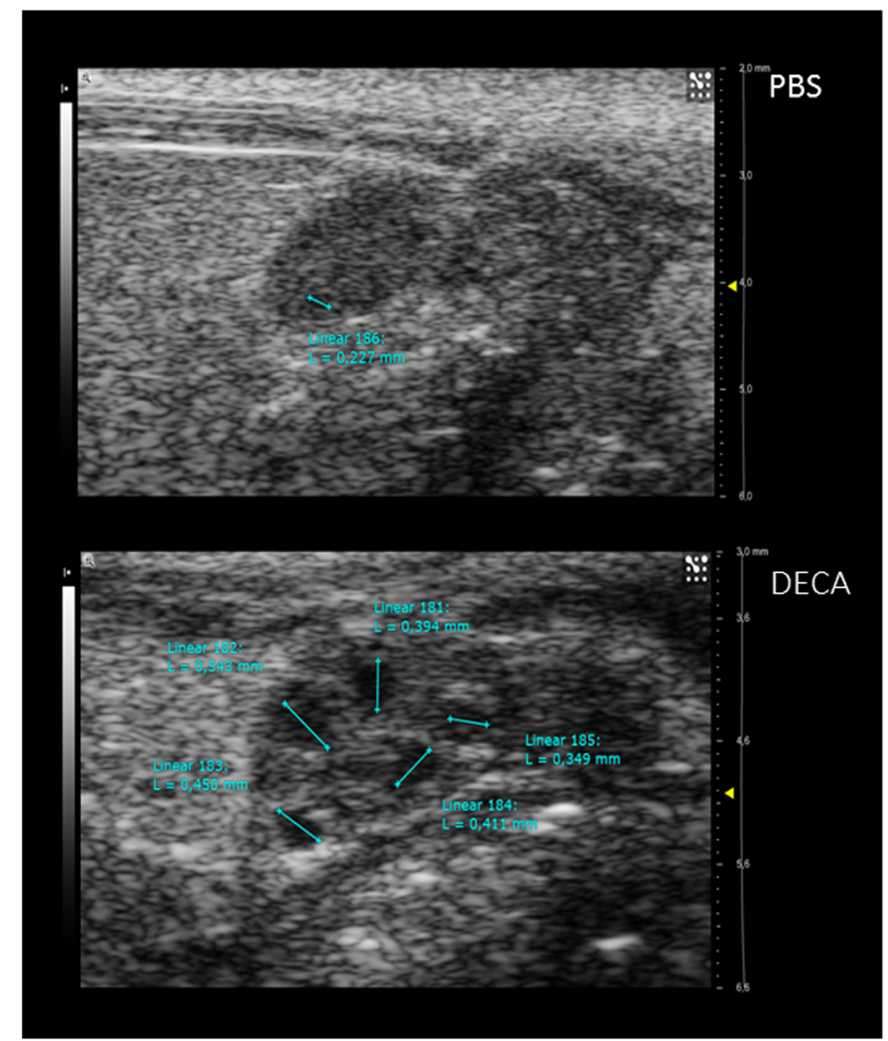

Dominant follicle diameter

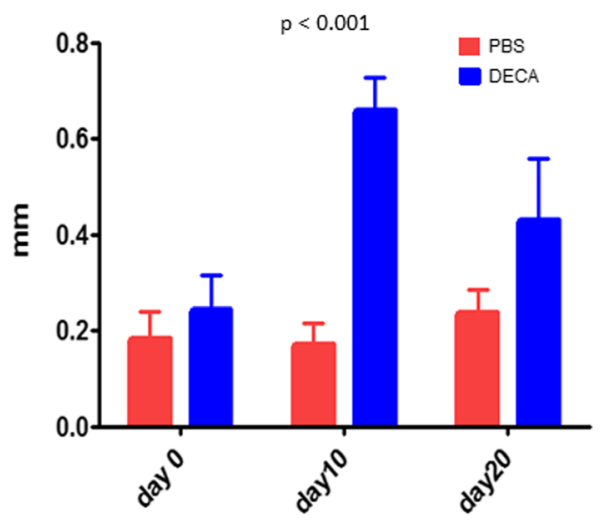

Fig. 3 US images (arrows) show the increase in the diameter of the dominant follicle in a decapeptyl-treated mouse (DECA) compared with a control mouse, which received only phosphate-buffered saline (PBS). The graph shows the comparison between the dominant follicle diameter of PBS-treated mice and that of decapeptyl-treated mice 

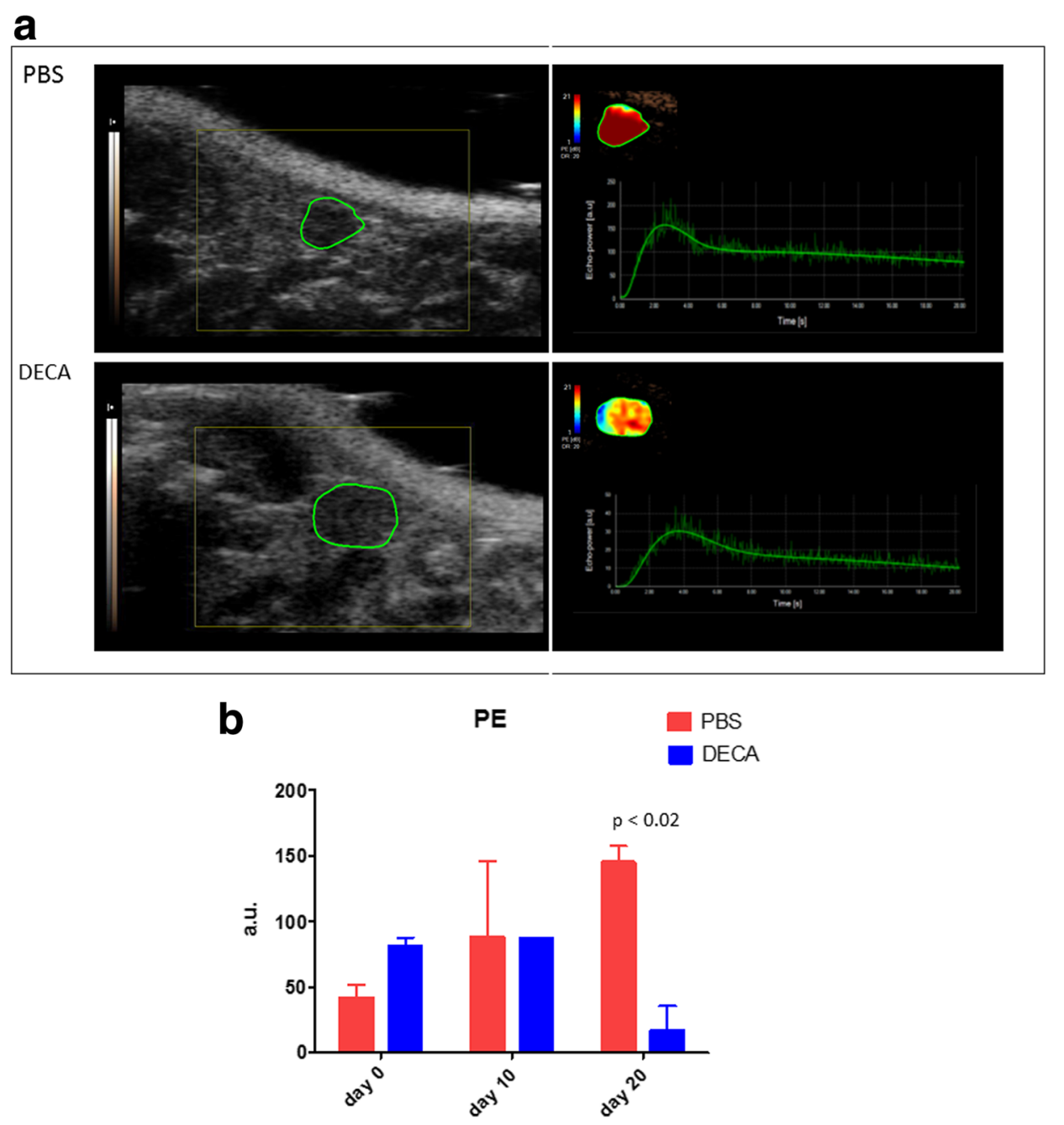

Wi AUC

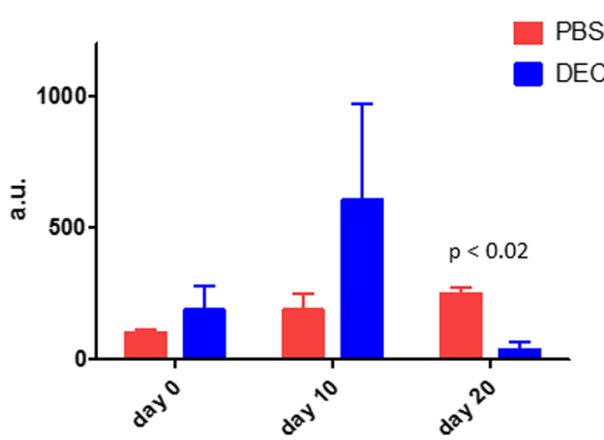

Fig. 4 a Contrast-enhanced ultrasound shows a reduced ovary perfusion was found in a decapeptyl-treated mouse (DECA) compared with a control untreated mouse (PBS). For each image, the area circled in green shows the ovary. The right side shows the graph representation of the dynamic enhancement. b At day 20, significantly reduced peak enhancement (PE) and wash-in area under the curve (WiAUC) were found in $\mathrm{GnRH}$-a-treated mice (DECA) compared with control mice (PBS)

allowed in all cases a quick identification at US and also an optimal dynamic visualisation during CEUS acquisition. Two other technical issues were important for ensuring US/CEUS feasibility without any problem of acquisition/interpretation data, including perfusion parameter extraction and quantitative software analysis. They 
were the use of a high-definition US system (Vevo, VisualSonics) equipped with mechanical arm and cutting-edge technology for the study of small experimental animals and the prone position of the mice, eliminating any possible artefacts due to gas bowel. Of note, in one of the few CEUS experimental studies performed to detect doxorubicin-induced damage to gonadal blood vessels, simultaneously performed on ovarian, testicular, and femoral arteries with the mice in conventional supine position [17], ovarian blood flow measurements were affected by bowel movements, probably hampering data findings. In our series performed with mice in prone position, technical problems with US and CEUS were never found. This technical approach can potentially become the standard method to study the ovaries in the murine model, although further studies with a larger cohort of mice will be necessary.

Of nine available CEUS quantitative parameters, the peak enhancement and the wash-in rate showed significant differences between decapeptyl-treated and control mice. From these preliminary findings, decapeptyl-treated mice showed a significant increase in dominant follicle and a significant decrease in vascularisation. These data give new insight in elucidating the potential role of $\mathrm{GnRH}-\mathrm{a}$ in ovarian protection. GnRH-a administration seems not to impair folliculogenesis, as in treated mice the diameter of the dominant follicle was increased [18]. Interestingly, in our study, the decapeptyl-treated group showed a significant decrease in vasculature compared to controls. Previous studies have described that changes in ovarian blood flow are influenced by gonadotropin secretion, and it has already been reported how the protective of the GnRH-a in mice exposed to the toxic activity of cyclophosphamide could be mediated by a decline in ovarian blood flow and a resulting decrease in the exposure of the ovaries to the chemotherapy agent [19]. Hasky et al. [20] found a decrease in vascular endothelial growth factor in mice treated with doxorubicin and $\mathrm{GnRH}-\mathrm{a}$, compared to mice treated with doxorubicin alone.

In conclusion, our preliminary experimental study showed that the ovaries can be assessed with US and CEUS with mice placed in the prone position, providing quantitative information. This technical approach may be the standard method for US evaluation of the ovaries in the murine model. A significant increase in the diameter of the dominant follicle at US and a significant reduction in vascularisation at CEUS in decapeptyl-treated mice compared to controls were observed. This information will be useful for further prospective studies combining $\mathrm{GnRH}-\mathrm{a}$ and chemotherapy agents evaluating the effects on ovarian function to obtain more translational information useful for clinical practice.

\section{Abbreviations}

CEUS: Contrast-enhanced ultrasound; GnRH-a: Gonadotropin-releasing hormone analogue; US: Ultrasound
Availability of data and materials

Data and materials can be provided on request.

Funding

The authors state that this work has not received any funding.

Acknowledgements

The authors thank Ipsen for the collaboration.

Authors' contributions

$M V, A B, L P, A M S, M P, P V$, and GM contributed to study design, manuscript preparation, drafting, and revision; EGR and $A C$ to data collection, drafting, and revision; FDC, ADM, MC, CT, and AE to study design. All authors read and approved the final manuscript.

Ethics approval

The study was approved by the Animal Care and Use Committees of San Raffaele Hospital, Milan, Italy.

Consent for publication

Not applicable

Competing interests

Not applicable

\section{Publisher's Note}

Springer Nature remains neutral with regard to jurisdictional claims in published maps and institutional affiliations.

\section{Author details}

${ }^{1}$ Department of Radiology, San Raffaele Scientific Institute, Milan, Italy ¿Experimental Imaging Center, San Raffaele Scientific Institute, Milan, Italy. ${ }^{3}$ Obstetrics and Gynecology Unit, San Raffaele Scientific Institute, Milan, Italy.

${ }^{4}$ Vita-Salute San Raffaele University, Milan, Italy.

Received: 18 August 2018 Accepted: 26 October 2018

Published online: 19 December 2018

References

1. Suter MB, Pagani O (2018) Should age impact breast cancer management in young women? Fine tuning of treatment guidelines. Ther Adv Med Oncol 22:1758835918776923

2. Morgan S, Anderson RA, Gourley C, Wallace WH, Spears N (2012) How do chemotherapeutic agents damage the ovary? Hum Reprod Update 18:525-535

3. Bedoschi G, Navarro PA, Oktay K (2016) Chemotherapy-induced damage to ovary: mechanisms and clinical impact. Future Oncol 12:2333-2344

4. Oktay K, Harvey BE, Partridge AH et al (2018) Fertility preservation in patients with cancer: ASCO clinical practice guideline update. J Clin Oncol 36:1994-2001

5. Del Mastro L, Lambertini M (2017) Gonadotropin-releasing hormone analogs for ovarian function protection during chemotherapy in young early breast cancer patients: the last piece of the puzzle? Ann Oncol 28: 1683-1685

6. Lutz AM, Bachawal SV, Drescher CW, Pysz MA, Willmann JK, Gambhir SS (2014) Ultrasound molecular imaging in a human CD276 expressionmodulated murine ovarian cancer model. Clin Cancer Res 20:1313-1322

7. Needles A, Arditi M, Rognin NG et al (2010) Nonlinear contrast imaging with an array-based micro-ultrasound system. Ultrasound Med Biol 36:2097-2106

8. Lambertini M, Anserini P, Fontana $V$ et al (2017) The PREgnancy and FERtility (PREFER) study: an Italian multicenter prospective cohort study on fertility preservation and pregnancy issues in young breast cancer patients. BMC Cancer 17:346

9. Letourneau JM, Ebbel EE, Katz PP et al (2012) Acute ovarian failure underestimates age- specific reproductive impairment for young women undergoing chemotherapy for cancer. Cancer 118:1933-1939

10. Ataya K, Rao LV, Lawrence E, Kimmel R (1995) Luteinizing hormonereleasing hormone agonist inhibits cyclophosphamide-induced ovarian follicular depletion in rhesus monkeys. Biol Reprod 52:365-372

11. Tworoger SS, Fairfield KM, Colditz GA, Rosner BA, Hankinson SE (2007) Association of oral contraceptive use, other contraceptive methods, and infertility with ovarian cancer risk. Am J Epidemiol 166:894-901 
12. Massarotti C, Scaruffi P, Lambertini M, Remorgida V, Del Mastro L, Anserini P (2017) State of the art on oocyte cryopreservation in female cancer patients: a critical review of the literature. Cancer Treat Rev 57:50-57

13. Wong M, O'Neill S, Walsh G, Smith IE (2013) Goserelin with chemotherapy to preserve ovarian function in pre-menopausal women with early breast cancer: menstruation and pregnancy outcomes. Ann Oncol 24:133-138

14. Park HJ, Koo YA, Im YH, Yoon BK, Choi D (2010) GnRH agonist therapy to protect ovarian function in young Korean breast cancer patients. J Korean Med Sci 25:110-116

15. Munster PN, Moore AP, Ismail-Khan R et al (2012) Randomized trial using gonadotropin- releasing hormone agonist triptorelin for the preservation of ovarian function during (neo) adjuvant chemotherapy for breast cancer. J Clin Oncol 30:533-538

16. Moore HC, Unger JM, Phillips KA et al (2015) Goserelin for ovarian protection during breast-cancer adjuvant chemotherapy. N Engl J Med 372:923-932

17. Bar-Joseph H, Ben-Aharon I, Tzabari M, Tsarfaty G, Stemmer SM, Shalgi R (2011) In vivo bioimaging as a novel strategy to detect doxorubicin-induced damage to gonadal blood vessels. PLOS One 6:e23492

18. Detti L, Uhlmann RA, Zhang J et al (2014) Goserelin fosters bone elongation but does not prevent ovarian damage in cyclophosphamide-treated prepubertal mice. Fertil Steril 101:1157-1164

19. Meirow D, Assad G, Dor J, Rabinovici J (2004) The GnRH antagonist cetrorelix reduces cyclophosphamide-induced ovarian follicular destruction in mice. Hum Reprod 19:1294-1299

20. Hasky N, Uri-Belapolsky S, Goldberg K et al (2015) Gonadotrophin-releasing hormone agonists for fertility preservation: unraveling the enigma? Hum Reprod 30:1089-1101

\section{Submit your manuscript to a SpringerOpen ${ }^{\circ}$ journal and benefit from:}

- Convenient online submission

- Rigorous peer review

- Open access: articles freely available online

- High visibility within the field

- Retaining the copyright to your article

Submit your next manuscript at $\boldsymbol{\nabla}$ springeropen.com 